\title{
Dynamic Behaviors in a Droop Model for Phytoplankton Growth in a Chemostat with Nutrient Periodically Pulsed Input
}

\author{
Kai Wang, ${ }^{1}$ Zhidong Teng, ${ }^{2}$ and Xueliang Zhang ${ }^{1}$ \\ ${ }^{1}$ Department of Medical Engineering and Technology, Xinjiang Medical University, Urumqi 830054, China \\ ${ }^{2}$ College of Mathematics and System Sciences, Xinjiang University, Urumqi 830046, China
}

Correspondence should be addressed to Kai Wang, wangkaimath@sina.com

Received 23 December 2011; Accepted 20 March 2012

Academic Editor: Xue He

Copyright (C) 2012 Kai Wang et al. This is an open access article distributed under the Creative Commons Attribution License, which permits unrestricted use, distribution, and reproduction in any medium, provided the original work is properly cited.

The dynamic behaviors in a droop model for phytoplankton growth in a chemostat with nutrient periodically pulsed input are studied. A series of new criteria on the boundedness, permanence, extinction, existence of positive periodic solution and global attractivity for the model are established. Finally, an example is given to demonstrate the effectiveness of the results in this paper.

\section{Introduction}

The chemostat is a very important apparatus used to study the growth of microorganisms in a continuous cultured environment in a laboratory (see [1]). It may be viewed as a laboratory model of a simple lake with continuous stirring. Chemostat models have attracted widely the attention of the scientific community, since they have a wide range of applications, for example, waste-water treatment, production by genetically altered organisms (like production of insulin), and so forth. The growth in a chemostat is described by the systems of ordinary differential equations or functional differential equations. Many important and interesting results can be found in articles [2-10] and the references cited therein.

The droop model $[11,12]$ of phytoplankton growth is essential in theoretical phytoplankton ecology. The droop model takes into consideration that phytoplankton cells store nutrient and that the growth rate depends on the stored nutrient. Algae can uptake nutrient in excess of current needs and continue to grow during nutrient poor conditions. These nutrients are supplied from an external reservoir; in several earlier works, this concentration is assumed to be constant. The droop model of phytoplankton growth in a chemostat has been widely investigated in many literatures (see [13-19]). As in [16], the classical chemostat 
equations modeling phytoplankton population dynamics originally related the growth rate of the cells to the nutrient concentration in the medium. It is assumed that the nutrient uptake rate is proportional to the rate of reproduction. The constant of proportionality that converts units of nutrient to units of organisms is called the yield constant. Because of the assumed constant value of the yield, the classical Monod model is referred to as the constant-yield model in [15]. In [17], both uniform persistence and the existence of periodic coexistence state are established for a periodically forced droop model on two phytoplankton species competition in a chemostat under some appropriate conditions. In [18], the authors considered the nonautonomous droop model for phytoplankton growth in a chemostat in which the nutrient input varies nonperiodically. It is assumed that growth rate varies with the internal nutrient level of the cell and the uptake rate of phytoplankton depends on both the external and the internal nutrient concentrations. A series of new criteria on the positivity, boundedness, permanence, and extinction of the population is established.

This work was motivated by [19], where the droop model was presented with general functions for growth and uptake, which takes the following forms:

$$
\begin{gathered}
\frac{d S(t)}{d t}=D\left(S^{0}(t)-S\right)-N(t) \rho(S, Q), \\
\frac{d Q(t)}{d t}=\rho(S, Q)-\mu(Q) Q, \\
\frac{d N(t)}{d t}=N(\mu(Q)-D) .
\end{gathered}
$$

The author proved that the periodically forced droop model has precisely two dynamic regimes depending on a threshold condition involving the dilution rate. If the dilution rate is such that the subthreshold condition hold, the phytoplankton population is washed out of the chemostat. If the superthreshold condition holds, then there is a unique periodic solution to which all solutions approach asymptotically.

As well known, countless organisms live in seasonally or diurnally forced environment, in which the populations obtain food, so the effects of this forcing may be quite profound. Recently, many papers studied chemostat model with variations in the supply of nutrients or the washout. The chemostat models with impulsive input perturbation have been studied in many articles, see [20-30] and the references cited therein, where many important and interesting results on the persistence, permanence and extinction of microorganisms, global stability, the existence of periodic oscillation and dynamical complexity of the systems are discussed.

However, we find that, up to now, few papers consider the droop model with nutrient periodically pulsed input. From the above discussion, this subject has broad biological significance. In pulse case, we will give some good extensions of the corresponding results given by Smith in [19]. We introduce the following droop model for phytoplankton growth in a chemostat with nutrient periodically pulsed input

$$
\begin{gathered}
\frac{d S(t)}{d t}=-D S-N(t) \rho(S, Q), \\
\frac{d Q(t)}{d t}=\rho(S, Q)-\mu(Q) Q, \quad t \neq n T, \\
\frac{d N(t)}{d t}=N(\mu(Q)-D),
\end{gathered}
$$




$$
\begin{gathered}
S\left(t^{+}\right)=S(t)+p, \\
Q\left(t^{+}\right)=Q(t), \quad t=n T, n \in N, \\
N\left(t^{+}\right)=N(t) .
\end{gathered}
$$

For model (1), we will investigate the permanence and extinction of the species, and the existence of positive $T$-periodic solution and global attractivity of the model. We also will give an example and numerical simulations to demonstrate the effectiveness of the results in this paper.

This paper is organized as follows. In the following section we will firstly introduce some basic assumption for model (1). Furthermore, we also will give several useful lemmas. In Section 3, we will state and prove an ultimate boundedness theorem of solutions for model (1). In Section 4, we will state and prove the sufficient condition on the permanence of species for model (1). In Section 5, the sufficient condition on the extinction of species for model (1) is given. In Section 6, the existence of positive $T$-periodic solution and the global attractivity of solutions for model (1) are established. Finally, in Section 7, we will discuss an example and give some numerical simulations.

\section{Preliminaries}

In model (1), $N(t)$ denotes the phytoplankton cells at biomass concentration, $S(t)$ denotes the nutrient at concentration in a growth medium. Each phytoplankton cell is assumed to possess an internal pool of stored nutrient, also called quota $Q(t) . D>0$ is the input and output flow, and its referred to as the dilution rate; $p>0$ is the amount of the substrate concentration pulsed each $n T$, where $T>0$ is a constant. Function $\mu(Q)$ denotes the phytoplankton growth rate, function $\rho(S, Q)$ denotes the nutrient uptake rate. In this paper, for model (1), we always assume that the following condition holds.

$\left(H_{1}\right)$ Function $\mu(Q)$ is continuously differentiable and $d \mu(Q) / d Q>0$ for all $Q \geq Q_{\text {min }}$, $\mu\left(Q_{\min }\right)=0$, where $Q_{\min }$ is some given positive constant.

$\left(H_{2}\right)$ Function $\rho(S, Q)$ is continuously differentiable and $\partial \rho(S, Q) / \partial S>0$, $\partial \rho(S, Q) / \partial Q \leq 0$ for all $S>0$ and $Q>Q_{\min }$. Further, $\rho(0, Q) \equiv 0$ for all $Q \geq Q_{\text {min }}$.

The initial condition in model (1) is given in the following form:

$$
S\left(0^{+}\right)=S_{0}>0, \quad Q\left(0^{+}\right)=Q_{0} \geq Q_{\text {min }}, \quad N\left(0^{+}\right)=N_{0}>0 .
$$

Firstly, on the positivity of solutions for model (1), we have the following result.

Lemma 2.1. Any solution $(S(t), Q(t), N(t))$ of model (1) with initial condition (2.1) is positive, that is, $S(t)>0, Q(t) \geq Q_{\min }$ and $N(t)>0$ for all $t>0$.

Proof. Obviously, for any $t>0$, we have

$$
N(t)=N\left(0^{+}\right) \exp \int_{0}^{t}(\mu(Q(s))-D) d s>0 .
$$


We consider $S(t)$. If the conclusion is not true, then from $S\left(0^{+}\right)>0$ and $p>0$ we can obtain that there is a $t_{1}>0$ such that $S\left(t_{1}\right)=0$ and $S(t)>0$ for all $t \in\left(0, t_{1}\right)$. From the mean-value theorem and assumption $\left(H_{2}\right)$, for any $t \in\left(0, t_{1}\right)$, there is a $\xi(t) \in(0, S(t))$ such that

$$
\rho(S(t), Q(t))=\frac{\partial \rho(\xi(t), Q(t))}{\partial S} S(t)
$$

Hence, from the first equation of model (1), we obtain

$$
\frac{d S(t)}{d t}=-\left(D+N(t) \frac{\partial \rho(\xi(t), Q(t))}{\partial S}\right) S(t) \quad \forall t \in\left(0, t_{1}\right], t \neq n T
$$

Choose an integer $n_{0} \geq 0$ such that $t_{1} \in\left(n_{0} T,\left(n_{0}+1\right) T\right]$. Obviously, $S\left(n_{0} T^{+}\right)>0$ and integrating (2.4) from $n_{0} T$ to $t_{1}$,

$$
S\left(t_{1}\right)=S\left(n_{0} T^{+}\right) \exp \left(-\int_{n_{0} T}^{t_{1}}\left(D+N(u) \frac{\partial \rho(\xi(u), Q(u))}{\partial S}\right)\right) d u>0,
$$

which leads to a contradiction.

Now, we consider $Q(t)$. We have $Q\left(0^{+}\right) \geq Q_{\min }$ and $\mu\left(Q_{\min }\right)=0$. If $Q\left(0^{+}\right)=Q_{\min }$, then since right derivative

$$
\left.\frac{d Q(t)}{d t}\right|_{t=0^{+}}=\rho\left(S\left(0^{+}\right), Q_{\min }\right)>0
$$

there is a $t_{1}>0$ such that $Q(t)>Q_{\min }$ for all $t \in\left(0, t_{1}\right]$. If there is a $t_{2}>t_{1}$ such that $Q\left(t_{2}\right)=Q_{\min }$ and $Q(t)>Q_{\min }$ for all $t \in\left(0, t_{2}\right)$, then left derivative $d Q(t) /\left.d t\right|_{t=t_{2}^{-}}$. But from assumption $\left(H_{2}\right)$, we have

$$
\left.\frac{d Q(t)}{d t}\right|_{t=t_{2}^{-}}=\rho\left(S\left(t_{2}\right), Q_{\min }\right)>0
$$

which leads to a contradiction. If $Q\left(0^{+}\right)>Q_{\min }$, then similarly to the above, we also can obtain a contradiction. This completes the proof of Lemma 2.1.

Now, we consider the following linear impulsive differential equation

$$
\begin{aligned}
& \frac{d \omega(t)}{d t}=-D \omega(t)-\eta, \quad t \neq n T, \\
& \omega\left(t^{+}\right)=\omega(t)+p, \quad t=n T, n \in N,
\end{aligned}
$$

where $D$ and $p$ are defined in model (1) and $\eta$ is a constant. Clearly,

$$
\omega^{*}(t)=-\frac{\eta}{D}+S^{*}(t), \quad t \in(n T,(n+1) T], n \in N
$$


is the $T$-periodic solution of $(2.8)$, where

$$
S^{*}(t)=\frac{p e^{-D(t-n T)}}{1-e^{-D T}}, \quad t \in(n T,(n+1) T], \quad n \in N
$$

We say that $\omega^{*}(t)$ is globally uniformly attractive if for any constants $M>0$ and $\varepsilon>0$ there is a constant $T(M, \varepsilon)>0$ such that for any initial time $t_{0} \geq 0$ and initial value $\omega_{0}$ with $\left|\omega_{0}\right| \leq M$, one has

$$
\left|\omega\left(t, t_{0}, \omega_{0}\right)-\omega^{*}(t)\right|<\varepsilon \quad \forall t \geq t_{0}+T(M, \varepsilon)
$$

where $\omega\left(t, t_{0}, \omega_{0}\right)$ is the solution of (2.8) with initial condition $\omega\left(t_{0}\right)=\omega_{0}$. We have following result.

Lemma 2.2 (see [27]). T-periodic solution $\omega^{*}(t)$ of (2.8) is globally uniformly attractive.

For (2.8), when $\eta=0$, we obtain the subsystem of model (1) with $N(t)=0$ as follows:

$$
\begin{gathered}
\frac{d S(t)}{d t}=-D S(t), \quad t \neq n T, \\
S\left(t^{+}\right)=S(t)+p, \quad t=n T, n \in N .
\end{gathered}
$$

Clearly, $S^{*}(t)$ is the positive $T$-periodic solution of (2.12). From Lemma 2.2, we obtain that for any solution $S(t)$ of $(2.12)$, one has

$$
S(t)-S^{*}(t) \longrightarrow 0 \quad \text { as } t \longrightarrow \infty
$$

Putting $S(t)=S^{*}(t)$ in the second equation of model (1), we obtain

$$
\frac{d Q(t)}{d t}=\rho\left(S^{*}(t), Q(t)\right)-\mu(Q(t)) Q(t)
$$

From the expression of $S^{*}(t)$, we can choose a constant $0<\alpha_{0}<p e^{-D T} /\left(1-e^{-D T}\right)$ such that

$$
\inf _{t \geq 0} S^{*}(t)-\alpha_{0} \geq \frac{p e^{-D T}}{1-e^{-D T}}-\alpha_{0}>0 .
$$

For any $\alpha \in\left[-\alpha_{0}, \alpha_{0}\right]$, we consider the following equation:

$$
\frac{d Q(t)}{d t}=\rho\left(S^{*}(t)+\alpha, Q(t)\right)-\mu(Q(t)) Q(t)
$$

For any initial points $t_{0} \geq 0$ and $Q_{0} \geq Q_{\text {min }}$, let $Q_{0}(t)$ and $Q_{\alpha}(t)$ be the solutions of (2.14) and (2.16) satisfying initial conditions $Q_{0}\left(t_{0}\right)=Q_{0}$ and $Q_{\alpha}\left(t_{0}\right)=Q_{0}$, respectively. We have the following result. 
Lemma 2.3. (a) There are constants $M_{1}>1$ and such that for any $t_{0} \geq 0, Q_{0} \geq Q_{\min }$ and $\alpha \in$ $\left[-\alpha_{0}, \alpha_{0}\right]$

$$
Q_{\min } \leq \liminf _{t \rightarrow \infty} Q_{\alpha}(t) \leq \liminf _{t \rightarrow \infty} Q_{\alpha}(t) \leq M_{1}
$$

(b) For each $\alpha \in\left[-\alpha_{0}, \alpha_{0}\right]$, (2.16) has a positive and globally uniformly attractive T-periodic solution $Q_{\alpha}^{*}(t)$.

(c) $Q_{\alpha}^{*}(t)$ converges to $Q_{0}^{*}(t)$ uniformly for $t \in[0, T]$ as $\alpha \rightarrow 0$.

Proof. Firstly, inequality $Q(t) \geq Q_{\min }$ for all $t \in[0, \infty)$ can be proved by using the similar argument as in the proof of Lemma 2.1.

From assumptions $\left(H_{1}\right)$ and $\left(H_{2}\right)$, there are positive constants $K$ and $\delta$ such that for any $\alpha \in\left[-\alpha_{0}, \alpha_{0}\right]$

$$
\int_{0}^{T}\left[\frac{\rho\left(S^{*}(u)+\alpha, K\right)}{K}-\mu(K)\right] d u<-\delta
$$

If $Q_{\alpha}(t) \geq K$ for all $t \geq t_{0}$, then integrating (2.16), for any $t \geq t_{0}$, we have

$$
\begin{aligned}
Q_{\alpha}(t) & =Q_{\alpha}\left(t_{0}\right) \exp \int_{t_{0}}^{t}\left[\frac{\rho\left(S^{*}(u)+\alpha, Q_{\alpha}(u)\right)}{Q_{\alpha}(u)}-\mu\left(Q_{\alpha}(u)\right)\right] d u \\
& \leq Q_{\alpha}\left(t_{0}\right) \exp \int_{t_{0}}^{t}\left[\frac{\rho\left(S^{*}(u)+\alpha, K\right)}{K}-\mu(K)\right] d u \\
& \leq Q_{\alpha}\left(t_{0}\right) \exp \left[-\delta\left(t-t_{0}\right)\right] .
\end{aligned}
$$

Hence, $Q_{\alpha}(t) \rightarrow 0$ as $t \rightarrow \infty$, which leads to a contradiction. Therefore, there is a $t_{1}>t_{0}$ such that $Q_{\alpha}\left(t_{1}\right)<K$. Further, if there are $t_{2}>t_{3}>t_{1}$ such that $Q_{\alpha}\left(t_{2}\right)>K \exp \left(M^{*} T\right), Q_{\alpha}\left(t_{3}\right)=K$, and $Q_{\alpha}(t)>K$ for all $t \in\left(t_{3}, t_{2}\right]$, where

$$
M^{*}=\sup _{t \in R_{+}}\left\{\frac{\rho\left(S^{*}(t)+\alpha_{0}, K\right)}{K}+\mu(K)\right\}
$$

then we can choose an integer $q \geq 0$ such that $t_{2} \in\left(t_{3}+q T, t_{3}+(q+1) T\right]$ and integrating (2.16) to obtain

$$
\begin{aligned}
K \exp \left(M^{*} T\right) & <Q_{\alpha}\left(t_{2}\right) \\
& =Q_{\alpha}\left(t_{3}\right) \exp \int_{t_{3}}^{t_{2}}\left[\frac{\rho\left(S^{*}(u)+\alpha, Q_{\alpha}(u)\right)}{Q_{\alpha}(u)}-\mu\left(Q_{\alpha}(u)\right)\right] d u \\
& \leq K \exp \left(\int_{t_{3}}^{t_{2}+q T}+\int_{t_{3}+q T}^{t_{2}}\right)\left[\frac{\rho\left(S^{*}(u)+\alpha, K\right)}{K}-\mu(K)\right] d u \\
& \leq K \exp \int_{t_{3}+q T}^{t_{2}}\left[\frac{\rho\left(S^{*}(u)+\alpha, K\right)}{K}-\mu(K)\right] d u \\
& \leq K \exp \left(M^{*} T\right)
\end{aligned}
$$


which also leads to a contradiction. Therefore, we finally have

$$
Q_{\alpha}(t) \leq K \exp \left(M^{*} T\right) \quad \forall t \geq t_{1} .
$$

Choose constant $M_{1}=K \exp \left(M^{*} T\right)$, then we have $\limsup _{t \rightarrow \infty} Q_{\alpha}(t) \leq M_{1}$ for all $\alpha \in$ $\left[-\alpha_{0}, \alpha_{0}\right]$. Thus, conclusion $(a)$ is proved.

From conclusion (a), directly using the main results given by Teng and Chen in [31], we can obtain that (2.16) for each $\alpha \in\left[-\alpha_{0}, \alpha_{0}\right]$ has a positive $T$-periodic solution $Q_{\alpha}^{*}(t)$.

For any constant $\eta_{0}>1$ and $t_{0} \geq 0$, let $Q_{\alpha}(t)$ be a solution of (2.16) with initial value $Q_{\alpha}\left(t_{0}\right) \in\left[\eta_{0}^{-1}, \eta_{0}\right]$. By conclusion $(a)$, there is a constant $M_{1}>1$ such that

$$
M_{1}^{-1} \leq Q_{\alpha}^{*}(t) \leq M_{1}, \quad M_{1}^{-1} \leq Q_{\alpha}(t) \leq M_{1} \quad \forall t \geq t_{0} .
$$

Consider Liapunov function

$$
V(t)=\left|\ln Q_{\alpha}(t)-\ln Q_{\alpha}^{*}(t)\right|
$$

Calculating the Dini derivative $D^{+} V(t)$, from

$$
\operatorname{sign}\left(Q_{\alpha}(t)-Q_{\alpha}^{*}(t)\right)\left[\frac{\rho\left(S^{*}(t)+\alpha, Q_{\alpha}(t)\right)}{Q_{\alpha}(t)}-\frac{\rho\left(S^{*}(t)+\alpha, Q_{\alpha}^{*}(t)\right)}{Q_{\alpha}^{*}(t)}\right] \leq 0,
$$

we can obtain for any $t \geq t_{0}$ and $t \neq n T$

$$
D^{+} V(t) \leq \operatorname{sign}\left(Q_{\alpha}(t)-Q_{\alpha}^{*}(t)\right)\left[\mu\left(Q_{\alpha}^{*}(t)\right)-\mu\left(Q_{\alpha}(t)\right)\right] .
$$

Using the mean-value theorem, we further obtain

$$
D^{+} V(t) \leq-\frac{d \mu(\xi(t))}{d Q}\left|Q_{\alpha}^{*}(t)-Q_{\alpha}(t)\right|
$$

where $\xi(t)$ is situated between $Q_{\alpha}^{*}(t)$ and $Q_{\alpha}(t)$. When $t=n T$ and $n \in N$, we obviously have $V\left(t^{+}\right)=V(t)$. Hence

$$
V(t) \leq V\left(t_{0}\right) \quad \forall t \geq t_{0}
$$

Consequently, by (2.23) we have

$$
\left|\ln Q_{\alpha}(t)\right| \leq\left|\ln Q_{\alpha}^{*}(t)\right|+V\left(t_{0}\right) \leq \ln \left(\eta_{0} M_{1}^{2}\right),
$$

for all $t \geq t_{0}$. Hence

$$
\eta_{0}^{-1} M_{1}^{-2} \leq Q_{\alpha}(t) \leq \eta_{0} M_{1}^{2} \quad \text { for all } t \geq t_{0}
$$


Further, from (2.23) we obtain

$$
\eta_{0}^{-1} M_{1}^{-2} V(t) \leq\left|Q_{\alpha}^{*}(t)-Q_{\alpha}(t)\right| \leq \eta_{0} M_{1}^{2} V(t)
$$

for all $t \geq t_{0}$. Consequently, from (2.27) it follows that

$$
D^{+} V(t) \leq-\eta_{0}^{-1} M_{1}^{-2} \frac{d \mu(\xi(t))}{d Q} V(t) \leq-M_{0} V(t)
$$

where

$$
M_{0}=\eta_{0}^{-1} M_{1}^{-2} \min \left\{\frac{d \mu(\xi(t))}{d Q}: \eta_{0}^{-1} M_{1}^{-2} \leq Q \leq \eta_{0} M_{1}^{2}\right\}
$$

Hence, we further have

$$
V(t) \leq V\left(t_{0}\right) e^{-M_{0}\left(t-t_{0}\right)} \leq \ln \left(\eta_{0} M_{1}\right) e^{-M_{0}\left(t-t_{0}\right)}, \quad \forall t \geq t_{0}
$$

For any constant $\varepsilon>0$, from (2.34), choose

$$
T\left(\eta_{0}, \varepsilon\right)=-\frac{1}{M_{0}} \ln \frac{\varepsilon}{\eta_{0} M_{1}^{2} \ln \left(\eta_{0} M_{1}\right)}
$$

then for any $t \geq t_{0}+T\left(\eta_{0}, \varepsilon\right)$ we can obtain

$$
V(t)<\frac{\varepsilon}{\eta_{0} M_{1}^{2}} \quad \forall t \geq t_{0}+T\left(\eta_{0}, \varepsilon\right) .
$$

Therefore,

$$
\left|Q_{\alpha}^{*}(t)-Q_{\alpha}(t)\right|<\varepsilon \quad \forall t \geq t_{0}+T\left(\eta_{0}, \varepsilon\right) .
$$

This shows solution $Q_{\alpha}^{*}(t)$ is globally uniformly attractive. Thus, conclusion $(b)$ is proved.

Finally, we prove conclusion $(c)$. From assumptions $\left(H_{1}\right)$ and $\left(H_{2}\right)$, we can easily prove that the right hand of model (1) satisfies the uniform Lipschitz condition with respect to $\alpha \in\left[-\alpha_{0}, \alpha_{0}\right]$. Hence, by the continuity of solutions with respect to parameter $\alpha$, we can obtain that $Q_{\alpha}^{*}(t) \rightarrow Q_{0}^{*}(t)$ uniformly for $t \in[0, T]$ as $\alpha \rightarrow 0$. Thus, conclusion (c) is proved. This completes the proof of Lemma 2.3. result.

When $\alpha=0$, (2.16) degenerates into (2.14), from Lemma 2.3 we have the following

Corollary 2.4. Equation (2.14) has a unique positive T-periodic solution $Q_{0}^{*}(t)$ which is globally uniformly attractive. 
The following lemma will be used in the proof of the result on the global attractivity of model (1).

Lemma 2.5. Let function $f(t)$ be continuous on $[0,+\infty)$ and differentiable for any $t \neq n T(n \in N)$, where $T>0$ is a constant. If there exist a constant $M>0$, such that $|d f(t) / d t|<M$ for any $t \neq n T(n \in N)$, then $f(t)$ is uniformly continuous on $[0,+\infty)$.

The proof of Lemma 2.1 is simple, we hence omit it here.

\section{Boundedness}

On the ultimate boundedness of all positive solutions of model (1), we have the following result.

Theorem 3.1. Let $(S(t), Q(t), N(t))$ be any positive solution of model (1), then we have that there exists a constant $M_{3}>0$, which is independent of any positive solution of model (1), such that

$$
\limsup _{t \rightarrow \infty} S(t)<M_{3}, \quad \limsup _{t \rightarrow \infty} Q(t)<M_{3}, \quad \limsup _{t \rightarrow \infty} N(t)<M_{3} .
$$

Proof. Let $(S(t), Q(t), N(t))$ be any positive solution of model (1) with initial condition (2.1). Since

$$
\begin{aligned}
& \frac{d S(t)}{d t} \leq-D S(t), \quad t \neq n T, \\
& S\left(t^{+}\right)=S(t)+p, \quad t=n T, n \in N,
\end{aligned}
$$

by Lemma 2.2 we directly have

$$
\limsup _{t \rightarrow \infty} S(t) \leq \limsup _{t \rightarrow \infty} S^{*}(t) \leq \frac{p}{1-e^{-D T}} .
$$

For any constant $\varepsilon>0$, there is a $T_{0}>0$ such that

$$
S(t) \leq \frac{p}{1-e^{-D T}}+\varepsilon \quad \forall t \geq T_{0}
$$

From the second equation of model (1), we have

$$
\frac{d Q(t)}{d t} \leq \rho\left(\frac{p}{1-e^{-D T}}+\varepsilon, Q(t)\right)-\mu(Q(t)) Q(t)
$$

for all $t \geq T_{0}$. Using the similar argument as in the proof of Lemma 2.3, we have that there is a positive constant $M_{Q}>0$, and $M_{Q}$ is independent of any solution $(S(t), Q(t), N(t))$ of model (1), such that

$$
\limsup _{t \rightarrow \infty} Q(t) \leq M_{Q}
$$


Define a function $U(t)$ as follows:

$$
U(t)=S(t)+Q(t) N(t)
$$

Calculating the derivative of $U(t)$ along solution $(S(t), Q(t), N(t))$ of model (1), we have

$$
\begin{gathered}
\frac{d U(t)}{d t}=-D U(t), \quad t \neq n T, \\
U\left(t^{+}\right)=U(t)+p, \quad t=n T, \quad n \in N .
\end{gathered}
$$

From Lemma 2.2, we obtain

$$
\limsup _{t \rightarrow \infty} U(t) \leq \limsup _{t \rightarrow \infty} S^{*}(t) \leq \frac{p}{1-e^{-D T}}
$$

From Lemma 2.1, we have $Q(t) \geq Q_{\min }$ for all $t \geq 0$. Hence, by (3.9) we can obtain that $N(t)$ is ultimately bound. This completes the proof of Theorem 3.1.

Remark 3.2. Obviously, Theorem 3.1 is new and can serve as an extension of corresponding result given by Smith in [19].

\section{Permanence}

On the permanence of species for model (1), we have the following result.

Theorem 4.1. Suppose

$$
\int_{0}^{T}\left[\mu\left(Q_{0}^{*}(t)\right)-D\right] d t>0
$$

Then model (1) is permanent.

Proof. From Lemma 2.1 we directly have $\liminf _{t \rightarrow \infty} Q(t) \geq Q_{\min }>0$. Hence, $Q(t)$ is obviously permanent. We now prove the permanence of $N(t)$. From (4.1), we can choose a constant $\varepsilon_{0}>0$ small enough such that

$$
\int_{0}^{T}\left[\mu\left(Q_{0}^{*}(t)-\varepsilon_{0}\right)-D\right] d t>\varepsilon_{0}
$$

Let $(S(t), Q(t), N(t))$ be any solution of model (1) with initial condition (2.1). From Theorem 3.1, for any constant $\varepsilon>0$ there is a $T_{0}>0$ such that

$$
S(t) \leq M_{3}+\varepsilon, \quad Q(t) \leq M_{3}+\varepsilon, \quad N(t) \leq M_{3}+\varepsilon
$$


for all $t \geq T_{0}$. For any $\alpha \in\left[-\alpha_{0}, \alpha_{0}\right]$, we consider the following assistant equation:

$$
\frac{d Q(t)}{d t}=\rho\left(S^{*}(t)+\alpha, Q(t)\right)-\mu(Q(t)) Q(t) .
$$

By Lemma 2.3, we obtain that (4.4) has a unique positive $T$-periodic solution $Q_{\alpha}^{*}(t)$, which is globally uniformly attractive and $Q_{\alpha}^{*}(t)$ converges to $Q_{0}^{*}(t)$ uniformly for $t \in R_{+}$as $\alpha \rightarrow 0$. Hence, there is an $\alpha^{*}>0$ and $\alpha^{*}<\varepsilon_{0}$ such that

$$
Q_{\alpha}^{*}(t)>Q_{0}^{*}(t)-\frac{\varepsilon_{0}}{2} \quad \forall t \in R_{+},|\alpha|<\alpha^{*}
$$

Choose positive constants $\varepsilon_{1}$ and $\varepsilon_{2}$ such that

$$
\begin{gathered}
\inf _{t \in R_{+}} S^{*}(t)-\varepsilon_{2}-\frac{\varepsilon_{1} \rho\left(M_{3}+\varepsilon, Q_{\text {min }}\right)}{D}>0, \\
\varepsilon_{2}+\frac{\varepsilon_{1} \rho\left(M_{3}+\varepsilon, Q_{\text {min }}\right)}{D}<\alpha^{*} .
\end{gathered}
$$

We first proof that

$$
\lim _{t \rightarrow \infty} \sup N(t)>\varepsilon_{1}
$$

for any solution $(S(t), Q(t), N(t))$ of model (1) with initial condition (2.1).

In fact, if (4.8) is not true, then there is a solution $(S(t), Q(t), N(t))$ of model (1) such that $\lim \sup _{t \rightarrow \infty} N(t) \leq \varepsilon_{1}$. Hence, there is a $T_{1}>T_{0}$ such that $N(t)<\varepsilon_{1}$, for all $t \geq T_{1}$. From the fist equation of model (1) and (4.3), we have

$$
\begin{gathered}
\frac{d S(t)}{d t} \geq-D S(t)-\varepsilon_{1} \rho\left(M_{3}+\varepsilon, Q_{\min }\right), \quad t \geq T_{1}, \quad t \neq n T \\
S\left(t^{+}\right)=S(t)+p, \quad t=n T, \quad n \in N
\end{gathered}
$$

From the comparison theorem of impulsive differential equations and Lemma 2.2, for above $\varepsilon_{2}>0$, there is an $n_{1} \in N$ and $n_{1} T>T_{1}$ such that

$$
S(t) \geq-\frac{\varepsilon_{1} \rho\left(M_{3}+\varepsilon, Q_{\min }\right)}{D}+S^{*}(t)-\varepsilon_{2} \quad \forall t>n_{1} T .
$$

From the second equation of model (1), we have

$$
\frac{d Q(t)}{d t} \geq \rho\left(S^{*}(t)-\frac{\varepsilon_{1} \rho\left(M_{3}+\varepsilon, Q_{\min }\right)}{D}-\varepsilon_{2}, Q(t)\right)-\mu(Q(t)) Q(t)
$$


for all $t>n_{1} T$. Let $\alpha_{1}=-\varepsilon_{1} \rho\left(M_{3}+\varepsilon, Q_{\text {min }}\right) / D-\varepsilon_{2}$, from Lemma 2.3 and the comparison theorem, there exists an $n_{2}>n_{1}$ such that

$$
Q(t) \geq Q_{\alpha_{1}}^{*}(t)-\frac{\varepsilon_{0}}{2} \geq Q_{0}^{*}(t)-\varepsilon_{0} \quad \forall t>n_{2} T
$$

Then, from the third equation of model (1), we have

$$
\frac{d N(t)}{d t} \geq N(t)\left(\mu\left(Q_{0}^{*}(t)-\varepsilon_{0}\right)-D\right) \quad \forall t>n_{2} T
$$

Integrating (4.13) from $n_{2} T$ to $t>n_{2} T$, we obtain

$$
N(t) \geq N\left(n_{2} T\right) \exp \int_{n_{2} T}^{t}\left(\mu\left(Q_{0}^{*}(s)-\varepsilon_{0}\right)-D\right) d s
$$

Obviously, from (4.2) and (4.14), we obtain $N(t) \rightarrow \infty$ as $t \rightarrow \infty$, which leads to a contradiction. Therefore, (4.8) is true.

Now, we prove that there is a constant $m_{1}>0$ such that

$$
\liminf _{t \rightarrow \infty} N(t) \geq m_{1}
$$

for any solution $(S(t), Q(t), N(t))$ of model (1) with initial condition (2.1).

Assume that (4.15) is not true, then there exists a sequence of initial values $z_{k}=$ $\left(S_{k}, Q_{k}, N_{k}\right)$, which satisfy $S_{k}>0, Q_{k} \geq Q_{\min }$, and $N_{k}>0$ such that for solution $\left(S\left(t, z_{k}\right), Q\left(t, z_{k}\right), N\left(t, z_{k}\right)\right)$ of model (1) with initial condition $S\left(0^{+}, z_{k}\right), Q\left(0^{+}, z_{k}\right)$, and $N\left(0^{+}, z_{k}\right)$

$$
\liminf _{t \rightarrow \infty} N\left(t, z_{k}\right)<\frac{\varepsilon_{1}}{k^{2}} \quad \forall k=1,2, \ldots
$$

From (4.8) and (4.16), we obtain that there exist two sequences $\left\{v_{q}^{(k)}\right\}$ and $\left\{t_{q}^{(k)}\right\}$ such that for each $k=1,2, \ldots$

$$
\begin{gathered}
0<v_{1}^{(k)}<t_{1}^{(k)}<v_{2}^{(k)}<t_{2}^{(k)}<\cdots<v_{q}^{(k)}<t_{q}^{(k)}<\cdots, \\
v_{q}^{(k)} \longrightarrow \infty, \quad v_{q}^{(k)} \longrightarrow \infty \quad \text { as } q \longrightarrow \infty, \\
N\left(t_{q}^{(k)}, z_{k}\right)=\frac{\varepsilon_{1}}{k^{2}}, \quad N\left(v_{q}^{(k)}, z_{k}\right)=\frac{\varepsilon_{1}}{k} \\
\frac{\eta}{k^{2}}<N\left(t, z_{k}\right)<\frac{\varepsilon_{1}}{k} \quad \forall t \in\left(v_{q}^{(k)}, t_{q}^{(k)}\right) .
\end{gathered}
$$

From Theorem 3.1, there is a $T^{(k)} \geq T_{0}$ such that

$$
S\left(t, z_{k}\right) \leq M_{3}+\varepsilon, \quad Q\left(t, z_{k}\right) \leq M_{3}+\varepsilon, \quad N\left(t, z_{k}\right) \leq M_{3}+\varepsilon
$$


for all $t \geq T^{(k)}$. Further for each $k$, from (4.17) there is an integer $K^{(k)}>0$ such that $v_{q}^{(k)}>T^{(k)}$ for all $q>K^{(k)}$. Hence for any $t \in\left[v_{q}^{(k)}, t_{q}^{(k)}\right]$ and $q>K^{(k)}$, we have

$$
\begin{aligned}
\frac{d N\left(t, z_{k}\right)}{d t} & =N\left(t, z_{k}\right)\left(\mu\left(Q\left(t, z_{k}\right)\right)-D\right) \\
& \geq-\gamma N\left(t, z_{k}\right),
\end{aligned}
$$

where $\gamma=D+\mu\left(M_{3}+\varepsilon\right)$. Therefore, for any $q \geq K^{(k)}$ and $k=1,2, \ldots$, integrating (4.21) on $\left[v_{q}^{(k)}, t_{q}^{(k)}\right]$, we obtain from $(4.18)$

$$
\begin{aligned}
\frac{\varepsilon_{1}}{k^{2}} & =N\left(t_{q}^{(k)}, z_{k}\right) \\
& \geq N\left(v_{q}^{(k)}, z_{k}\right) \exp \left[-\gamma\left(t_{q}^{(k)}-v_{q}^{(k)}\right)\right] \\
& =\frac{\varepsilon_{1}}{k} \exp \left[-\gamma\left(t_{q}^{(k)}-v_{q}^{(k)}\right)\right] .
\end{aligned}
$$

Consequently,

$$
t_{q}^{(k)}-v_{q}^{(k)} \geq \frac{\ln k}{r} \quad \forall q \geq K^{(k)}, \quad k=1,2, \ldots
$$

Consider the following equation:

$$
\begin{gathered}
\frac{d u(t)}{d t}=-D u(t)-\varepsilon_{1} \rho\left(M_{3}+\varepsilon_{0}, Q_{\min }\right), \quad t \neq n T, \\
u\left(t^{+}\right)=u(t)+p, \quad t=n T .
\end{gathered}
$$

From Lemma 2.2, there is a constant $T^{*}>0$ such that for any $t_{0} \geq 0$ and $0 \leq u_{0} \leq M_{3}+\varepsilon$

$$
u(t) \geq S^{*}(t)-\frac{\varepsilon_{1} \rho\left(M_{3}+\varepsilon, Q_{\min }\right)}{D}-\varepsilon_{2} \quad \forall t \geq t_{0}+T^{*},
$$

where $u(t)$ is the solution of (4.24) with initial value $u\left(t_{0}\right)=u_{0}$.

Further consider the following equation:

$$
\frac{d v(t)}{d t}=\rho\left(S^{*}(t)+\alpha_{1}, v(t)\right)-\mu(v(t)) v(t)
$$

From Lemma 2.3, there is a constant $T^{* *}>0$ such that for any $t_{0} \geq 0$ and $0 \leq v_{0} \leq M_{3}+\varepsilon$

$$
v(t) \geq Q_{\alpha_{1}}^{*}(t)-\frac{\varepsilon_{0}}{2} \quad \forall t \geq t_{0}+T^{* *},
$$

where $v(t)$ is the solution of (4.26) with initial value $v\left(t_{0}\right)=v_{0}$. 
From (4.23), we can choose an integer $N_{0}>0$ such that

$$
t_{q}^{(k)}-v_{q}^{(k)}>T^{*}+T^{* *}+T \quad \forall k>N_{0}, \quad q>K^{(k)} .
$$

Since for any integer $k, q \geq K^{(k)}, t \in\left[v_{q}^{(k)}, t_{q}^{(k)}\right]$ and $q \geq K^{(k)}$ we have

$$
\begin{aligned}
\frac{d S\left(t, z_{k}\right)}{d t} & =-D S\left(t, z_{k}\right)-N\left(t, z_{k}\right) \rho\left(S\left(t, z_{k}\right), Q\left(t, z_{k}\right)\right) \\
& \geq-D S\left(t, z_{k}\right)-\varepsilon_{1} \rho\left(M_{3}+\varepsilon, Q_{\min }\right), \quad t \neq n T, \\
S\left(t^{+}, z_{k}\right) & =S\left(t, z_{k}\right)+p, \quad t=n T,
\end{aligned}
$$

from the comparison theorem and inequality (4.25), we have

$$
S\left(t, z_{k}\right) \geq u(t) \geq-\frac{\varepsilon_{1} \rho\left(M_{3}+\varepsilon, Q_{\min }\right)}{D}+S^{*}(t)-\varepsilon_{2}=S^{*}(t)+\alpha_{1}
$$

for all $t \in\left[v_{q}^{(k)}+T^{*}, t_{q}^{(k)}\right]$ and $q \geq K^{(k)}$. Further, since

$$
\frac{d Q\left(t, z_{k}\right)}{d t} \geq \rho\left(S^{*}(t)+\alpha_{1}, Q\left(t, z_{k}\right)\right)-\mu\left(Q\left(t, z_{k}\right)\right) Q\left(t, z_{k}\right)
$$

for all $t \in\left[v_{q}^{(k)}+T^{*}, t_{q}^{(k)}\right]$ and $q \geq K^{(k)}$. From the comparison theorem and inequality (4.27),

$$
Q\left(t, z_{k}\right) \geq Q_{\alpha_{1}}^{*}(t)-\frac{\varepsilon_{0}}{2} \geq Q_{0}^{*}(t)-\varepsilon_{0}
$$

for all $t \in\left[v_{q}^{(k)}+T^{*}+T^{* *}, t_{q}^{(k)}\right]$ and $q \geq K^{(k)}$. Hence, for any $q \geq K^{(k)}, k \geq N_{0}$ and $t \in\left[t_{q}^{(k)}-T, t_{q}^{(k)}\right]$, we have

$$
\begin{aligned}
\frac{d N\left(t, z_{k}\right)}{d t} & =N\left(t, z_{k}\right)\left(\mu\left(Q\left(t, z_{k}\right)-D\right)\right) \\
& \geq N\left(t, z_{k}\right)\left(\mu\left(Q_{0}^{*}(t)-\varepsilon_{0}\right)-D\right)
\end{aligned}
$$

Integrating (4.33) from $t_{q}^{(k)}-T$ to $t_{q}^{(k)}$, by (4.19) we obtain

$$
\begin{aligned}
\frac{\varepsilon_{1}}{k^{2}} & =N\left(t_{q}^{(k)}, z_{k}\right) \\
& \geq N\left(t_{q}^{(k)}-T, z_{k}\right) \exp \int_{t_{q}^{(k)}-T}^{t_{q}^{(k)}}\left(\mu\left(Q_{0}^{*}(t)-\varepsilon_{0}\right)-D\right) d t \\
& \geq \frac{\varepsilon_{1}}{k^{2}} \exp \left(\varepsilon_{0}\right) \\
& >\frac{\varepsilon_{1}}{k^{2}}
\end{aligned}
$$


which is contradictory. This contradiction shows that (4.15) is true for any positive solution $(S(t), Q(t), N(t))$ of model (1).

Next, we prove that $S(t)$ in model (1) is permanent. Let $(S(t), Q(t), N(t))$ be any solution of model (1) with initial condition (2.1). From the assumption of $\rho(S, Q)$ and the mean-value theorem, for any $t \geq 0$, there exists a $\xi(t) \in(0, S(t))$ such that

$$
\frac{\rho(S(t), Q(t))}{S(t)}=\frac{\rho(S(t), Q(t))-\rho(0, Q(t))}{S(t)}=\frac{\partial \rho(\xi(t), Q(t))}{\partial S} .
$$

Further, from assumption $\left(H_{2}\right)$ and the boundedness of solution $(S(t), Q(t), N(t))$, there exists a constant $M_{s}>0$ such that

$$
\frac{\rho(S(t), Q(t))}{S(t)}=\frac{\partial \rho(\xi(t), Q(t))}{\partial S}<M_{S} \quad \forall t \geq 0 .
$$

From the first equation of model (1), we obtain

$$
\begin{aligned}
\frac{d S(t)}{d t} & =-D S(t)-N(t) \rho(S(t), Q(t)) \\
& \geq-\left(D+\left(M_{3}+\varepsilon\right) M_{S}\right) S(t),
\end{aligned}
$$

for all $t \geq T_{0}$ and $t \neq n T$, and

$$
S\left(t^{+}\right)=S(t)+p, \quad t=n T, n \in N
$$

Using the comparison theorem and Lemma 2.2, we can obtain

$$
\liminf _{t \rightarrow \infty} S(t) \geq \frac{p \exp \left(-\left(D+\left(M_{3}+\varepsilon\right) M_{s}\right) T\right)}{-\left(D+\left(M_{3}+\varepsilon\right) M_{s}\right) T}
$$

This shows that $S(t)$ is permanent. This completes the proof of Theorem 4.1.

\section{Extinction}

On the extinction of species of model (1), we have the following result.

Theorem 5.1. Suppose

$$
\int_{0}^{T}\left[\mu\left(Q_{0}^{*}(t)\right)-D\right] d t<0
$$

Then for any positive solution $(S(t), Q(t), N(t))$ of model (1)

$$
\lim _{t \rightarrow \infty} S(t)=S^{*}(t), \quad \lim _{t \rightarrow \infty} Q(t)=Q_{0}^{*}(t), \quad \lim _{t \rightarrow \infty} N(t)=0
$$


Proof. From (5.1), there exists a constant $\varepsilon_{0}>0$ such that

$$
\int_{0}^{T}\left[\mu\left(Q_{0}^{*}(t)+\varepsilon_{0}\right)-D\right] d t<0
$$

For any $\alpha \in\left[-\alpha_{0}, \alpha_{0}\right]$, we consider the following equation:

$$
\frac{d Q(t)}{d t}=\rho\left(S^{*}(t)+\alpha, Q(t)\right)-\mu(Q(t)) Q(t)
$$

By Lemma 2.3, we obtain that positive $T$-periodic solution $Q_{\alpha}^{*}(t)$ of (5.4) is globally uniformly attractive and $Q_{\alpha}^{*}(t)$ converges to $Q_{0}^{*}(t)$ uniformly for $t \in R_{+}$as $\alpha \rightarrow 0$. Hence, there is an $\alpha^{*}>0$ and $\alpha^{*}<\varepsilon_{0}$ such that

$$
Q_{\alpha}^{*}(t)<Q_{0}^{*}(t)+\varepsilon_{0} \quad \forall t \in R_{+},|\alpha|<\alpha^{*}
$$

Let $(S(t), Q(t), N(t))$ be any solution of model (1) with initial condition (2.1). Since

$$
\begin{aligned}
& \frac{d S(t)}{d t} \leq-D S(t), \quad t \neq n T, \\
& S\left(t^{+}\right)=S(t)+p, \quad t=n T,
\end{aligned}
$$

for any constant $0<\varepsilon_{1}<\alpha^{*}$, from Lemma 2.2, there is a $T_{0}>0$ such that

$$
S(t) \leq S^{*}(t)+\varepsilon_{1}, \quad t \geq T_{0}
$$

From the second equation of model (1), we have

$$
\frac{d Q(t)}{d t} \leq \rho\left(S^{*}(t)+\varepsilon_{1}, Q(t)\right)-\mu(Q(t)) Q(t) \quad \forall t \geq T_{0}
$$

From Lemma 2.3 and the comparison theorem, there exists a function $\beta(t): R_{+} \rightarrow R$ satisfying $\beta(t) \rightarrow 0$ as $t \rightarrow \infty$ such that

$$
Q(t) \leq Q_{\varepsilon_{1}}^{*}(t)+\beta(t) \leq Q_{0}^{*}(t)+\beta(t)+\varepsilon_{0} \quad \forall t \geq T_{0}
$$

Since $\lim _{t \rightarrow \infty} \beta(t)=0$, we can obtain

$$
\lim _{t \rightarrow \infty} \int_{t}^{t+T}\left[\mu\left(Q_{0}^{*}(t)+\beta(t)+\varepsilon_{0}\right)-D\right] d t=\int_{0}^{T}\left[\mu\left(Q_{0}^{*}(t)+\varepsilon_{0}\right)-D\right] d t<0
$$


Hence, there exist constants $\bar{\eta}>0$ and $T_{1}>T_{0}$ such that when $t \geq T_{1}$

$$
\int_{t}^{t+T}\left[\mu\left(Q_{0}^{*}(t)+\beta(t)+\varepsilon_{0}\right)-D\right] d t \leq-\bar{\eta}
$$

and $|\beta(t)|<1$.

For any $t \geq T_{1}$, from the third equation of model (1), we have

$$
\frac{d N(t)}{d t} \leq N(t)\left[\mu\left(Q_{0}^{*}(t)+\beta(t)+\varepsilon_{0}\right)-D\right] \quad \forall t \geq T_{1} .
$$

We choose an integer $r_{t} \geq 0$ such that $t \in\left(T_{1}+r_{t} T, T_{1}+\left(r_{t}+1\right) T\right]$. Then, integrating (5.12) from $T_{1}$ to $t$, we obtain

$$
\begin{aligned}
N(t) & \leq N\left(T_{1}\right) \exp \int_{T_{1}}^{t}\left[\mu\left(Q_{0}^{*}(t)+\beta(t)+\varepsilon_{0}\right)-D\right] d t \\
& \leq N\left(T_{1}\right) \exp \left\{\left(\int_{T_{1}}^{T_{1}+r_{t} T}+\int_{T_{1}+r_{t} T}^{t}\right)\left[\mu\left(Q_{0}^{*}(t)+\beta(t)+\varepsilon_{0}\right)-D\right] d t\right. \\
& \leq N\left(T_{1}\right) \exp \left(-\bar{\eta} r_{t}\right) \exp \left(\left[\mu\left(M_{1}+1+\varepsilon_{0}\right)-D\right] T\right) .
\end{aligned}
$$

Therefore, by (5.11) we have $N(t) \rightarrow 0$ as $t \rightarrow \infty$.

For any constant $\varepsilon>0$, there is a $T^{*}>0$ such that $S(t) \leq M_{3}+\varepsilon$ and $N(t) \leq \varepsilon$ for all $t>T^{*}$. When $t>T^{*}$, from model (1) we obtain

$$
\begin{gathered}
\frac{d S(t)}{d t} \leq-D S(t), \quad t \neq n T, \\
S\left(t^{+}\right)=S(t)+p, \quad t=n T, n \in N, \\
\frac{d S(t)}{d t} \geq-D S(t)-\varepsilon \rho\left(M_{3}+\varepsilon, Q_{\min }\right), \quad t \neq n T, \\
S\left(t^{+}\right)=S(t)+p, \quad t=n T, \quad n \in N .
\end{gathered}
$$

Using the comparison theorem and Lemma 2.2, we can obtain that there is a $T>T^{*}$ such that

$$
-\frac{\varepsilon \rho\left(M_{3}+\varepsilon, Q_{\min }\right)}{D}+S^{*}(t)-\varepsilon \leq S(t) \leq S^{*}(t)+\varepsilon
$$

for all $t \geq T$. From the arbitrariness of $\varepsilon$, we finally obtain

$$
S(t) \longrightarrow S^{*}(t) \quad \text { as } t \longrightarrow \infty
$$

Finally, from Lemma 2.3, we can easily obtain

$$
Q(t) \longrightarrow Q_{0}^{*}(t) \quad \text { as } t \longrightarrow \infty
$$

This completes the proof of Theorem 5.1. 
Remark 5.2. Obviously, system (1) has a semitrivial $T$-periodic solution $\left(S^{*}(t), Q_{0}^{*}(t), 0\right)$ at which microorganism culture fails. Theorem 5.1 shows that $T$-periodic solution $\left(S^{*}(t), Q_{0}^{*}(t), 0\right)$ of system (1) is global attractivity.

Remark 5.3. The biological meaning of Theorems 3.1-5.1 is very significant, which can be seen in [18] (see Remarks 3 and 5 in [18]).

Remark 5.4. We notice that it is quite difficult to obtain the sufficient and necessary conditions on the extinction and permanence of species $N$. So, we take this problem in the future.

\section{Global Attractivity}

Now, we discuss the global attractivity of all positive solutions and the existence of positive $T$-periodic solution of model (1); we have the following result.

Theorem 6.1. Suppose inequality (4.1) holds. Then model (1) has a unique positive T-periodic solution, which is globally attractive.

Proof. Define Liapunov function $W(t)$ as follows:

$$
W(t)=S(t)+Q(t) N(t)
$$

From the proof of Theorem 3.1, we have $W(t) \rightarrow S^{*}(t)$ as $t \rightarrow \infty$. Hence, $W(t)=S^{*}(t)+\alpha(t)$, where $\alpha(t)$ is defined on $R^{+}$with $\lim _{t \rightarrow \infty} \alpha(t)=0$. Substitute $S(t)=S^{*}(t)+\alpha(t)-Q(t) N(t)$ into model (1), we obtain

$$
\begin{gathered}
\frac{d Q(t)}{d t}=\rho\left(S^{*}(t)+\alpha(t)-Q(t) N(t), Q(t)\right)-\mu(Q(t)) Q(t), \\
\frac{d N(t)}{d t}=N(t)(\mu(Q(t))-D) .
\end{gathered}
$$

Further, let $u(t)=Q(t) N(t)$; then system (6.2) changes into the following form:

$$
\begin{gathered}
\frac{d u(t)}{d t}=u(t)\left[\frac{\rho\left(S^{*}(t)+\alpha(t)-u(t), Q(t)\right)}{Q(t)}-D\right], \\
\frac{d Q(t)}{d t}=\rho\left(S^{*}(t)+\alpha(t)-u(t), Q(t)\right)-\mu(Q(t)) Q(t) .
\end{gathered}
$$

Let $\left(u_{1}(t), Q_{1}(t)\right)$ and $\left(u_{2}(t), Q_{2}(t)\right)$ be any two positive solutions of system (6.3), for any constant $\epsilon_{0}>0$ with $\underline{m}-\epsilon_{0}>0$, from Theorems 4.1 and 5.1, there exits a constant $T_{0}>0$ such that

$$
\underline{m}-\epsilon_{0} \leq Q_{i}(t) \leq M_{3}+\epsilon_{0}, \quad\left(\underline{m}-\epsilon_{0}\right)^{2} \leq u_{i}(t) \leq\left(M_{3}+\epsilon_{0}\right)^{2}, \quad i=1,2,
$$

for all $t \geq T_{0}$. Define the Liapunov function as follows:

$$
V(t)=\left|\ln u_{1}(t)-\ln u_{2}(t)\right|+\left|\ln Q_{1}(t)-\ln Q_{2}(t)\right| .
$$


Obviously, $V\left(t^{+}\right)=V(t)$ for all $t=n T$ and $n \in N$. Calculating the Dini derivative of $V(t)$, for any $t \geq T_{0}$ and $t \neq n T$, we have

$$
\begin{aligned}
D^{+} V(t)= & \operatorname{sign}\left(u_{1}(t)-u_{2}(t)\right) \\
& \times\left[\frac{\rho\left(S^{*}(t)+\alpha(t)-u_{1}(t), Q_{1}(t)\right)}{Q_{1}(t)}-\frac{\rho\left(S^{*}(t)+\alpha(t)-u_{2}(t), Q_{2}(t)\right)}{Q_{2}(t)}\right] \\
& +\operatorname{sign}\left(Q_{1}(t)-Q_{2}(t)\right) \\
& \times\left[\frac{\rho\left(S^{*}(t)+\alpha(t)-u_{1}(t), Q_{1}(t)\right)}{Q_{1}(t)}-\frac{\rho\left(S^{*}(t)+\alpha(t)-u_{2}(t), Q_{2}(t)\right)}{Q_{2}(t)}\right. \\
& \left.+\mu\left(Q_{2}(t)\right)-\mu\left(Q_{1}(t)\right)\right] \\
= & \left(\operatorname{sign}\left(u_{1}(t)-u_{2}(t)\right)+\operatorname{sign}\left(Q_{1}(t)-Q_{2}(t)\right)\right) \\
& \times\left[\frac{\rho\left(S^{*}(t)+\alpha(t)-u_{1}(t), Q_{1}(t)\right)}{Q_{1}(t)}-\frac{\rho\left(S^{*}(t)+\alpha(t)-u_{2}(t), Q_{2}(t)\right)}{Q_{2}(t)}\right] \\
& -\frac{d \mu(\xi(t))}{d Q}\left|Q_{1}(t)-Q_{2}(t)\right|,
\end{aligned}
$$

where $\xi(t)$ is situated between $Q_{1}(t)$ and $Q_{2}(t)$. We claim that

$$
\begin{gathered}
\left(\operatorname{sign}\left(u_{1}(t)-u_{2}(t)\right)+\operatorname{sign}\left(Q_{1}(t)-Q_{2}(t)\right)\right) \\
\times\left[\frac{\rho\left(S^{*}(t)+\alpha(t)-u_{1}(t), Q_{1}(t)\right)}{Q_{1}(t)}-\frac{\rho\left(S^{*}(t)+\alpha(t)-u_{2}(t), Q_{2}(t)\right)}{Q_{2}(t)}\right] \leq 0 .
\end{gathered}
$$

In fact, there are the following several possible cases:

(1) $u_{1}(t)<u_{2}(t), Q_{1}(t)<Q_{2}(t)$,

(2) $u_{1}(t)<u_{2}(t), Q_{1}(t)=Q_{2}(t)$,

(3) $u_{1}(t)<u_{2}(t), Q_{1}(t)>Q_{2}(t)$,

(4) $u_{1}(t)=u_{2}(t), Q_{1}(t)<Q_{2}(t)$,

(5) $u_{1}(t)=u_{2}(t), Q_{1}(t)=Q_{2}(t)$ ，

(6) $u_{1}(t)=u_{2}(t), Q_{1}(t)>Q_{2}(t)$,

(7) $u_{1}(t)>u_{2}(t), Q_{1}(t)<Q_{2}(t)$,

(8) $u_{1}(t)>u_{2}(t), Q_{1}(t)=Q_{2}(t)$,

(9) $u_{1}(t)>u_{2}(t), Q_{1}(t)>Q_{2}(t)$.

We only need to prove case (1). Cases (2)-(8) can be easily proved and case (9) can be proved similarly to case (1). For case (1), we have

$$
\operatorname{sign}\left(u_{1}(t)-u_{2}(t)\right)+\operatorname{sign}\left(Q_{1}(t)-Q_{2}(t)\right)=-2
$$


From assumption $\left(\mathrm{H}_{2}\right)$, we have

$$
\begin{aligned}
\frac{\rho\left(S^{*}(t)+\alpha(t)-u_{1}(t), Q_{1}(t)\right)}{Q_{1}(t)}-\frac{\rho\left(S^{*}(t)+\alpha(t)-u_{2}(t), Q_{2}(t)\right)}{Q_{2}(t)} \\
=\frac{\rho\left(S^{*}(t)+\alpha(t)-u_{1}(t), Q_{1}(t)\right)}{Q_{1}(t)}-\frac{\rho\left(S^{*}(t)+\alpha(t)-u_{2}(t), Q_{1}(t)\right)}{Q_{1}(t)} \\
+\frac{\rho\left(S^{*}(t)+\alpha(t)-u_{2}(t), Q_{1}(t)\right)}{Q_{1}(t)}-\frac{\rho\left(S^{*}(t)+\alpha(t)-u_{2}(t), Q_{2}(t)\right)}{Q_{2}(t)} \\
\geq \frac{\rho\left(S^{*}(t)+\alpha(t)-u_{2}(t), Q_{1}(t)\right)}{Q_{1}(t)}-\frac{\rho\left(S^{*}(t)+\alpha(t)-u_{2}(t), Q_{2}(t)\right)}{Q_{2}(t)} \\
\geq 0 .
\end{aligned}
$$

Hence, (6.7) is true. Therefore,

$$
D^{+} V(t) \leq-\frac{d \mu(\xi(t))}{d Q}\left|Q_{1}-Q_{2}\right| \leq-A\left|Q_{1}-Q_{2}\right|
$$

for all $t \geq T_{0}$ and $t \neq n T$, where

$$
A=\inf \left\{\frac{d \mu(Q(t))}{d Q}: \underline{m}-\epsilon_{0} \leq Q(t) \leq M_{3}+\epsilon_{0}\right\} .
$$

For any $t \geq T_{0}$, integrating from $T_{0}$ to $t$, we have

$$
V(t)+\int_{T_{0}}^{t} A\left|Q_{1}(s)-Q_{2}(s)\right| d s \leq V\left(T_{0}\right) .
$$

Hence,

$$
\int_{T_{0}}^{\infty}\left|Q_{1}(s)-Q_{2}(s)\right| d s<\infty .
$$

From Theorem 3.1 and system (6.3), we easily see that $d Q_{i}(t) / d t(i=1,2)$ are bounded for $t \geq T_{0}$ and $t \neq n T$. Since $\left|Q_{1}(t)-Q_{2}(t)\right|$ is continuous for all $t \geq T_{0}$, by Lemma 2.5, $\left|Q_{1}(t)-Q_{2}(t)\right|$ is uniformly continuous on $\left[T_{0}, \infty\right)$. Therefore, from Barbalat lemma, we obtain

$$
\lim _{t \rightarrow \infty}\left(Q_{1}(t)-Q_{2}(t)\right)=0 .
$$

Let

$$
\begin{gathered}
G(S, Q)=\frac{\rho(S, Q)}{Q}, \\
\alpha=\inf \left\{\frac{\partial \rho(S, Q)}{\partial S}: S \in\left[\underline{m}-\epsilon_{0}, M_{3}+\epsilon_{0}\right], Q \in\left[\underline{m}-\epsilon_{0}, M_{3}+\epsilon_{0}\right]\right\}, \\
\beta=\sup \left\{\left|\frac{\partial G(S, Q)}{\partial Q}\right|: S \in\left[\underline{m}-\epsilon_{0}, M_{3}+\epsilon_{0}\right], Q \in\left[\underline{m}-\epsilon_{0}, M_{3}+\epsilon_{0}\right]\right\} .
\end{gathered}
$$


From assumption $\left(H_{2}\right)$, we have $\alpha>0$ and $\beta>0$. Further, we consider the following Liapunov function:

$$
V_{1}(t)=\left|\ln u_{1}(t)-\ln u_{2}(t)\right|
$$

Obviously, $V_{1}\left(t^{+}\right)=V_{1}(t)$ for all $t=n T$ and $n \in N$. Calculating the Dini derivative of $V_{1}(t)$, for any $t \geq T_{0}$ and $t \neq n T$, from the mean-value theorem, we have

$$
\begin{aligned}
D^{+} V_{1}(t)= & \operatorname{sign}\left(u_{1}(t)-u_{2}(t)\right) \\
& \times\left[\frac{\rho\left(S^{*}(t)+\alpha(t)-u_{1}(t), Q_{1}(t)\right)}{Q_{1}}-\frac{\rho\left(S^{*}(t)+\alpha(t)-u_{2}(t), Q_{2}(t)\right)}{Q_{2}(t)}\right] \\
= & \operatorname{sign}\left(u_{1}(t)-u_{2}(t)\right) \\
& \times\left[\frac{\rho\left(S^{*}(t)+\alpha(t)-u_{1}(t), Q_{1}(t)\right)}{Q_{1}(t)}-\frac{\rho\left(S^{*}(t)+\alpha(t)-u_{2}(t), Q_{1}(t)\right)}{Q_{1}(t)}\right. \\
& \left.+\frac{\rho\left(S^{*}(t)+\alpha(t)-u_{2}(t), Q_{1}(t)\right)}{Q_{1}(t)}-\frac{\rho\left(S^{*}(t)+\alpha(t)-u_{2}(t), Q_{2}(t)\right)}{Q_{2}(t)}\right] \\
\leq & -\frac{\partial \rho\left(\xi_{1}(t), Q_{1}(t)\right)}{Q_{1}(t) \partial S}\left|u_{1}(t)-u_{2}(t)\right| \\
& +\left|\frac{\partial G\left(S^{*}(t)+\alpha(t)-u_{2}(t), \xi_{2}(t)\right)}{\partial Q}\right|\left|Q_{1}(t)-Q_{2}(t)\right| \\
\leq & -\alpha\left(\underline{m}-\epsilon_{0}\right)^{2}\left|\ln u_{1}(t)-\ln u_{2}(t)\right|+\delta(t) \\
= & -\alpha\left(\underline{m}-\epsilon_{0}\right)^{2} V_{1}(t)+\delta(t),
\end{aligned}
$$

where $\xi_{1}(t)$ is situated between $S^{*}(t)+\alpha(t)-u_{1}(t)$ and $S^{*}(t)+\alpha(t)-u_{2}(t), \xi_{2}(t)$ is situated between $Q_{1}(t)$ and $Q_{2}(t)$ and $\delta(t)=\beta\left|Q_{1}(t)-Q_{2}(t)\right|$. By the comparison theorem and the formula of variational constant for one-order linear differential equations, we can obtain

$$
V_{1}(t) \leq V\left(T_{0}\right) e^{-\alpha\left(\underline{m}-\varepsilon_{0}\right)^{2}\left(t-T_{0}\right)}+\int_{T_{0}}^{t} \delta(s) e^{\alpha\left(\underline{m}-\varepsilon_{0}\right)^{2}(s-t)} d s
$$

for all $t \geq T_{0}$. From $\lim _{t \rightarrow \infty} \delta(t)=0$, we easily obtain $V_{1}(t) \rightarrow 0$ as $t \rightarrow \infty$. Consequently,

$$
\lim _{t \rightarrow \infty}\left(u_{1}(t)-u_{2}(t)\right)=0
$$

For any two positive solutions $\left(S_{i}(t), Q_{i}(t), N_{i}(t)\right)(i=1,2)$, from $N_{i}(t)=u_{i}(t) / Q_{i}(t)$ and $S_{i}(t)=S^{*}(t)+\alpha(t)-Q_{i}(t) N_{i}(t)(i=1,2)$, we can further obtain

$$
\lim _{t \rightarrow \infty}\left(S_{1}(t)-S_{2}(t)\right)=0, \quad \lim _{t \rightarrow \infty}\left(N_{1}(t)-N_{2}(t)\right)=0 .
$$

This shows that all solutions of model (1) is globally attractive. 
Let $X(t, z)=(S(t, z), Q(t, z), N(t, z))$ be some positive solution of model (1) with initial condition $(S(0), Q(0), N(0))=z$, where $z=\left(z_{1}, z_{2}, z_{3}\right)$. From Theorems 3.1 and 5.1, there exist two constants $M>m>0$ such that

$$
\begin{aligned}
& m \leq \liminf _{t \rightarrow \infty} S(t, z) \leq \limsup _{t \rightarrow \infty} S(t, z) \leq M, \\
& m \leq \liminf _{t \rightarrow \infty} Q(t, z) \leq \limsup _{t \rightarrow \infty} Q(t, z) \leq M, \\
& m \leq \liminf _{t \rightarrow \infty} N(t, z) \leq \limsup _{t \rightarrow \infty} N(t, z) \leq M .
\end{aligned}
$$

Hence, there exists an integer $n_{0}>0$ such that sequence of vectors $\left\{X(n T, z) \in R^{3}: n \geq n_{0}\right\}$ is bounded. Hence, there exists a subsequence $\left\{n_{k}\right\}$ and a $z^{*} \in R^{3}$ such that

$$
\lim _{k \rightarrow \infty} X\left(n_{k} T, z\right)=z^{*}
$$

Let $X\left(t, z^{*}\right)=\left(S\left(t, z^{*}\right), Q\left(t, z^{*}\right), N\left(t, z^{*}\right)\right)$ be the solution of model (1) with initial condition $(S(0), Q(0), N(0))=z^{*}$. By the continuity of solutions of system (2.1) with respect to initial values, we obtain

$$
\lim _{k \rightarrow \infty} X\left(\left(n_{k}+1\right) T, z\right)=\lim _{k \rightarrow \infty} X\left(T, X\left(n_{k} T, z\right)\right)=X\left(T, z^{*}\right)
$$

On the other hand, we easily prove that $X(t+T, z)$ also is the solution of model (1). Hence, from the global attractivity of system (1) we can obtain

$$
\lim _{t \rightarrow \infty}(X(t, z)-X(t+T, z))=0
$$

Consequently,

$$
\lim _{k \rightarrow \infty}\left(X\left(n_{k} T, z\right)-X\left(\left(n_{k}+1\right) T, z\right)\right)=0 .
$$

Further from (6.22) and (6.23), we obtain $X\left(T, z^{*}\right)=z^{*}$. This shows that $X\left(t, z^{*}\right)$ is a positive $T$-periodic solution of model (1).

Finally, from above discussion we obtain that model (1) has a unique positive $T$ periodic solution, which is globally attractive. This completes the proof of Theorem 6.1.

Remark 6.2. According to Theorem 6.1, we can find that model (1) has a unique positive Tperiodic solution that is globally attractive as long as it is only permanent.

Remark 6.3. We easily find that Theorem 6.1 is a very good extension in pulse case of the corresponding results given by Smith in [19]. 


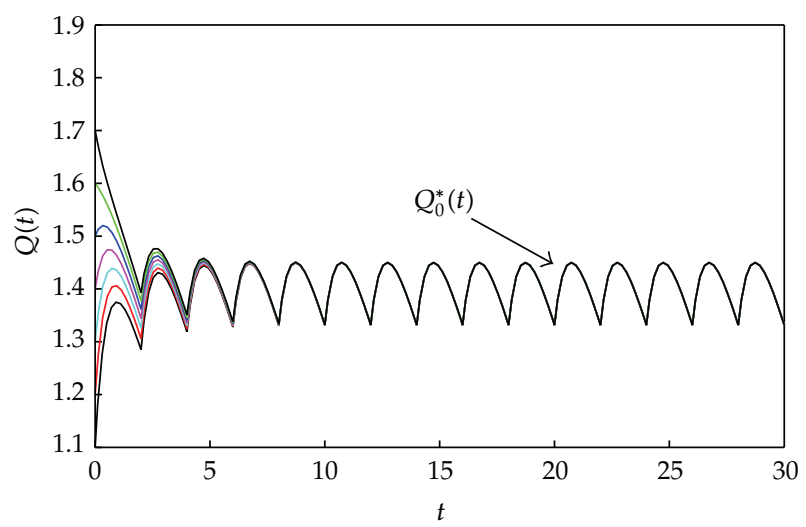

Figure 1: Time series of $Q_{0}^{*}(t)$.

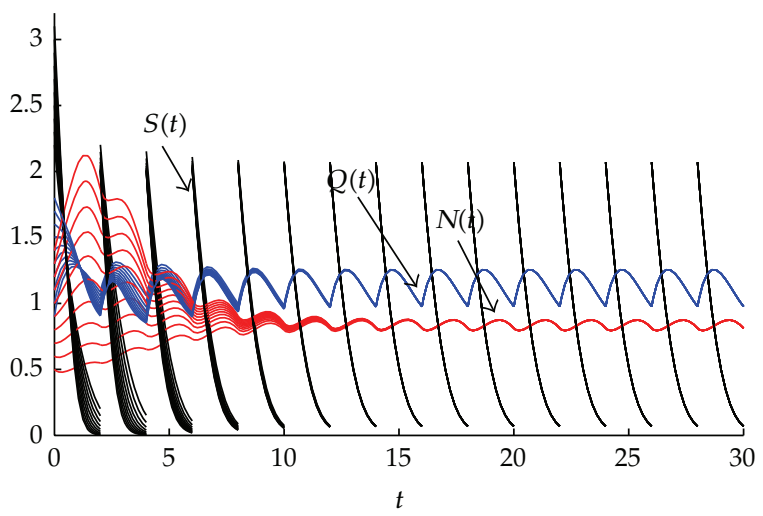

Figure 2: Dynamical behavior of system (1).

\section{Numerical Examples}

In this section, we will give an example to demonstrate the effectiveness of our main results. We consider model (1) with the following parameters:

$$
\mu(Q)=2\left(1-\frac{0.8}{Q}\right), \quad \rho(S, Q)=2 \frac{S}{1+S}, \quad p=2, \quad D=0.6, \quad T=2 .
$$

Obviously, assumptions $\left(H_{1}\right)$ and $\left(H_{2}\right)$ hold. We consider the following subsystem:

$$
\begin{gathered}
\frac{d S(t)}{d t}=-D S(t), \\
\frac{d Q(t)}{d t}=\rho(S(t), Q(t))-\mu(Q(t)) Q(t), \quad t \neq n T, \\
S\left(t^{+}\right)=S(t)+p, \quad t=n T, n \in N . \\
Q\left(t^{+}\right)=Q(t), \quad
\end{gathered}
$$


From Lemmas 2.2 and 2.3, system (7.2) has a periodic solution $\left(S^{*}(t), Q_{0}^{*}(t)\right)$. Further, let

$$
I=\int_{0}^{T}\left[\mu\left(Q_{0}^{*}(t)\right)-D\right] d t
$$

Since the precise expression of $Q_{0}^{*}(t)$ is quite complex, it is hard to determine the value of $I$. Therefore, here we will use the method of numerical simulation. Applying MatLab software, we can obtain the numerical simulation of $Q_{0}^{*}(t)$, see Figure 1 .

Furthermore, by numerical calculation, we can obtain

$$
\int_{0}^{T}\left[\mu\left(Q_{0}^{*}(t)\right)-D\right] d t \approx 0.93
$$

Therefore, we have $I>0$. Thus, by Theorem 6.1 we finally obtain that model (1) has a unique positive $T$-periodic solution, which is globally attractive, see Figure 2.

\section{Acknowledgments}

Supported by the National Natural Science Foundation of China (10961022) and Doctoral Scientific Research Fund of Xinjiang Medical University.

\section{References}

[1] H. L. Smith and P. Waltman, The Theory of the Chemostat, vol. 13 of Cambridge Studies in Mathematical Biology, Cambridge University Press, New York, NY, USA, 1995.

[2] E. Beretta and Y. Takeuchi, "Global stability for chemostat equations with delayed nutrient recycling," Nonlinear World, vol. 1, no. 3, pp. 291-306, 1994.

[3] G. J. Butler, S. B. Hsu, and P. Waltman, "A mathematical model of the chemostat with periodic washout rate," SIAM Journal on Applied Mathematics, vol. 45, no. 3, pp. 435-449, 1985.

[4] H. I. Freedman and Y. T. Xu, "Models of competition in the chemostat with instantaneous and delayed nutrient recycling," Journal of Mathematical Biology, vol. 31, no. 5, pp. 513-527, 1993.

[5] J. K. Hale and A. S. Somolinos, "Competition for fluctuating nutrient," Journal of Mathematical Biology, vol. 18, no. 3, pp. 255-280, 1983.

[6] X.-Z. He, S. Ruan, and H. Xia, "Global stability in chemostat-type equations with distributed delays," SIAM Journal on Mathematical Analysis, vol. 29, no. 3, pp. 681-696, 1998.

[7] Z. Li and R. Xu, "Stability analysis of a ratio-dependent chemostat model with time delay and variable yield," International Journal of Biomathematics, vol. 3, no. 2, pp. 243-253, 2010.

[8] J. M. Luo, S. L. Yuan, and W. G. Zhang, "Competition between two microorganisms in the chemostat with general variable yields and general growth rates," International Journal of Biomathematics, vol. 1, no. 4, pp. 463-474, 2008.

[9] Z. Q. Lu and J. J. Wu, "Global stability of chemostat model with delayed response in growth and a lethal external inhibitor," International Journal of Biomathematics, vol. 1, no. 4, pp. 503-520, 2008.

[10] S. S. Pilyugin and P. Waltman, "Competition in the unstirred chemostat with periodic input and washout," SIAM Journal on Applied Mathematics, vol. 59, no. 4, pp. 1157-1177, 1999.

[11] M. R. Droop, "Vitamin $B_{12}$ and marine ecology, IV: The kinetics of uptake, growth, and inhibition in Monochrysis ltheri," Journal of the Marine Biological Association of the United Kingdom, vol. 48, pp. 689-733, 1968.

[12] M. R. Droop, "Some thoughts on nutrient limitation in algae," Journal of Phycology, vol. 9, pp. 264-272, 1973.

[13] J. P. Grover, "Resource competition in a variable environment: phytoplankton growing according to the variable-internal-store model," The American Naturalist, vol. 138, pp. 811-835, 1991

[14] J. P. Grover, "Dynamics of competition among microalgae in variable environments: experimental tests of alternative models," Oikos, vol. 62, pp. 231-243, 1991. 
[15] J. P. Grover, "Constant and variable yield models of population growth: responses to environmental variability and implications for competition," Journal of Theoretical Biology, vol. 15, pp. 409-428, 1992.

[16] J. Monod, Recherches sur la Croissance des Cultures Bacteriennes, Hermann, Paris, France, 1942.

17] M. Pascual, "Periodic response to periodic forcing of the Droop equations for phytoplankton growth," Journal of Mathematical Biology, vol. 32, no. 8, pp. 743-759, 1994.

[18] M. Rehim and Z. Teng, "Mathematical analysis on nonautonomous droop model for phytoplankton growth in a chemostat: boundedness, permanence and extinction," Journal of Mathematical Chemistry, vol. 46, no. 2, pp. 459-483, 2009.

[19] H. L. Smith, "The periodically forced Droop model for phytoplankton growth in a chemostat," Journal of Mathematical Biology, vol. 35, no. 5, pp. 545-556, 1997.

[20] J. Jiao and L. Chen, "Dynamical analysis of a chemostat model with delayed response in growth and pulse input in polluted environment," Journal of Mathematical Chemistry, vol. 46, no. 2, pp. 502-513, 2009.

[21] X. Meng, Q. Zhao, and L. Chen, "Global qualitative analysis of new monod type chemostat model with delayed growth response and pulsed input in polluted environment," Applied Mathematics and Mechanics, vol. 29, no. 1, pp. 75-87, 2008.

[22] G. Pang, Y. Liang, and F. Wang, "Analysis of Monod type food chain chemostat with $k$-times periodically pulsed input," Journal of Mathematical Chemistry, vol. 43, no. 4, pp. 1371-1388, 2008.

[23] S. Sun and L. Chen, "Dynamic behaviors of Monod type chemostat model with impulsive perturbation on the nutrient concentration," Journal of Mathematical Chemistry, vol. 42, no. 4, pp. 837$847,2007$.

[24] Z. Teng, R. Gao, M. Rehim, and K. Wang, “Global behaviors of Monod type chemostat model with nutrient recycling and impulsive input," Journal of Mathematical Chemistry, vol. 47, no. 1, pp. 276-294, 2010.

[25] F. Wang, C. Hao, and L. Chen, "Bifurcation and chaos in a Tessiet type food chain chemostat with pulsed input and washout," Chaos, Solitons and Fractals, vol. 32, no. 4, pp. 1547-1561, 2007.

[26] F. Wang and G. Pang, "Competition in a chemostat with Beddington-DeAngelis growth rates and periodic pulsed nutrient," Journal of Mathematical Chemistry, vol. 44, no. 3, pp. 691-710, 2008.

[27] K. Wang, Z. Teng, and F. Zhang, "Global behaviors of a chemostat model with delayed nutrient recycling and periodically pulsed input," Discrete Dynamics in Nature and Society, vol. 2010, Article ID 830951, 21 pages, 2010.

[28] S. Zhang and D. Tan, "Study of a chemostat model with Beddington-DeAngelis functional response and pulsed input and washout at different times," Journal of Mathematical Chemistry, vol. 44, no. 1, pp. 217-227, 2008.

[29] Z. Zhao, L. Chen, and X. Song, "Extinction and permanence of chemostat model with pulsed input in a polluted environment," Communications in Nonlinear Science and Numerical Simulation, vol. 14, no. 4, pp. 1737-1745, 2009.

[30] X. Zhou, X. Song, and X. Shi, "Analysis of competitive chemostat models with the BeddingtonDeAngelis functional response and impulsive effect," Applied Mathematical Modelling, vol. 31, pp. 2299-2312, 2007.

[31] Z. D. Teng and L. S. Chen, “On the positive periodic solutions of periodic Kolmogorov type systems with delays," Acta Mathematicae Applicatae Sinica, vol. 22, no. 3, pp. 446-456, 1999 (Chinese). 


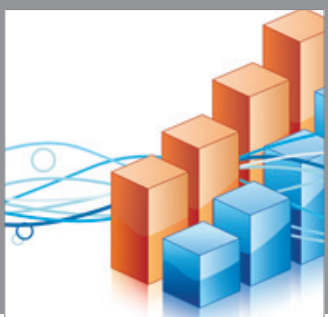

Advances in

Operations Research

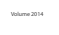

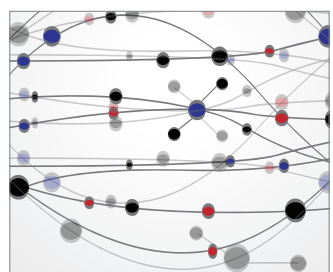

\section{The Scientific} World Journal
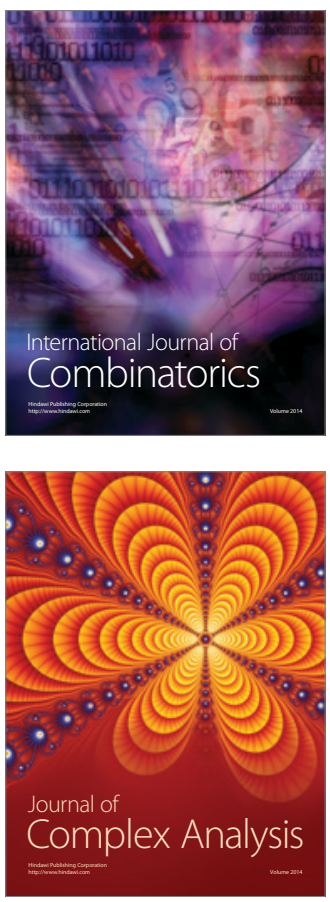

International Journal of

Mathematics and

Mathematical

Sciences
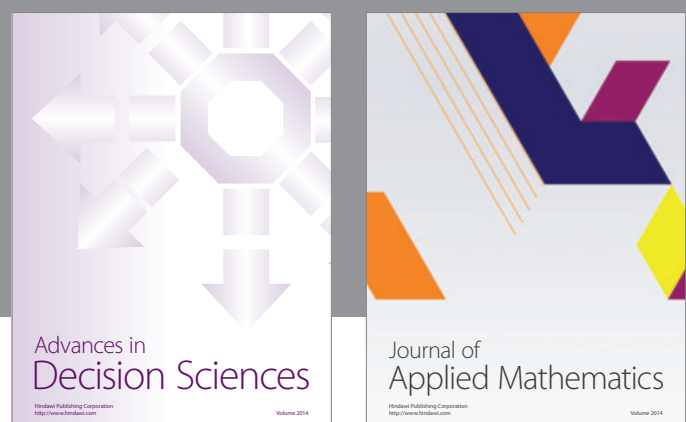

Journal of

Applied Mathematics
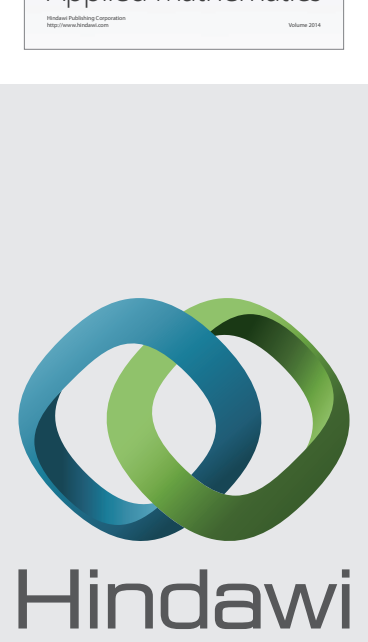

Submit your manuscripts at http://www.hindawi.com
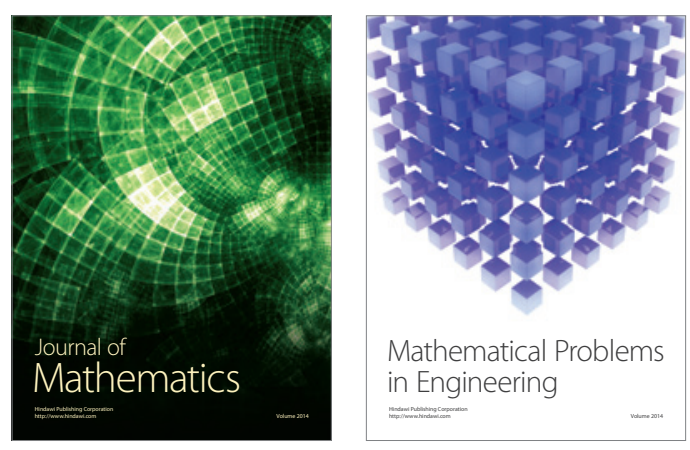

Mathematical Problems in Engineering
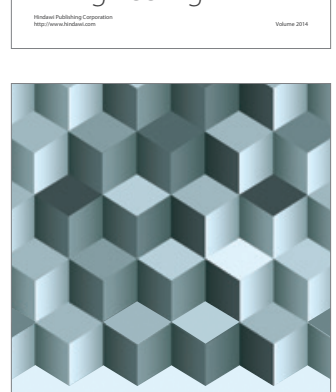

Journal of

Function Spaces
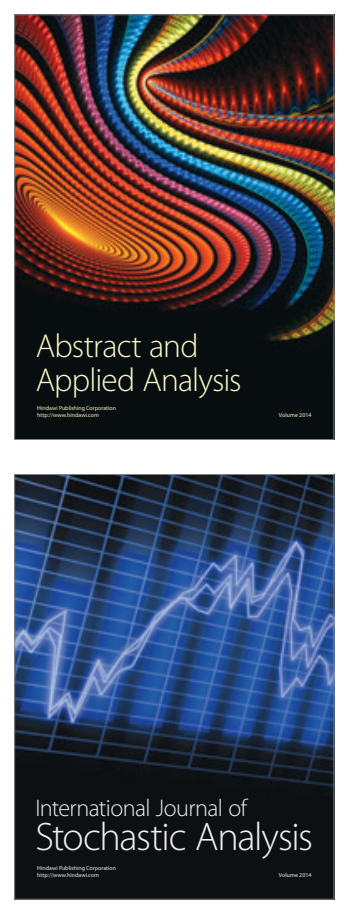

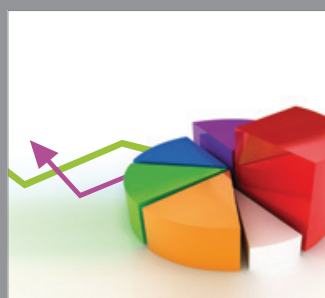

ournal of

Probability and Statistics

Promensencen
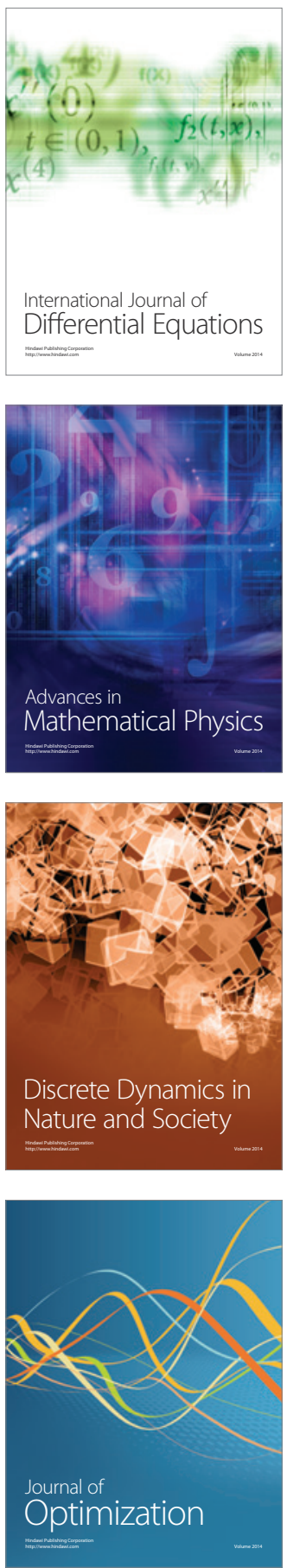